\title{
The use of Instanyl® in the treatment of breakthrough pain in cancer patients: a 3-month observational, prospective, cohort study
}

\author{
Ulf E. Kongsgaard • Martin Eeg • Hanna Greisen
}

Received: 18 September 2013 / Accepted: 13 January 2014 / Published online: 8 February 2014

(C) The Author(s) 2014. This article is published with open access at Springerlink.com

\begin{abstract}
Purpose Instanyl ${ }^{\circledR}$ (intranasal fentanyl spray) is a novel treatment for breakthrough pain (BTP) in cancer patients. It has shown a rapid onset of pain relief in clinical trials. This study examines the use of Instanyl ${ }^{\circledR}$ in real-life settings.

Methods A 3-month observational, prospective, cohort study of cancer patients with BTP receiving Instany $l^{\circledR}(50,100$, or $200 \mu \mathrm{g})$ under routine clinical practice. Data were collected at three time points corresponding with routine clinic visits baseline, Week 4, and Week 13. Primary outcomes: success of titration and maintenance dose after titration. Secondary outcomes: change in maintenance dose of Instanyl ${ }^{\circledR}$ and level of background pain medication; Brief Pain Inventory-Short Form (BPI-SF) and Patient Treatment Satisfaction Scale (PTSS) scores; adverse drug reactions (ADRs).

Results Titration with Instanyl ${ }^{\circledR}$ was successful in $84.5 \%$ of 309 patients; most patients were titrated at the lowest dose $(50 \mu \mathrm{g})$. The majority showed no change in maintenance dose, with little change in the level of background pain medication. BPI-SF and PTSS scores significantly improved from baseline to Week 4 . The main reason for terminating Instanyl ${ }^{\mathbb{R}}$ was death, as expected due to the underlying disease; incidence of ADRs was low and no fatal ADRs were reported.

Conclusions In a real-life group of cancer patients with disease progression, Instanyl ${ }^{\mathbb{R}}$ was titrated successfully at doses
\end{abstract}

U. E. Kongsgaard

Department of Anaesthesiology, Division of Emergencies and Critical Care, Oslo University Hospital and Medical Faculty,

University of Oslo, Oslo, Norway

M. Eeg $\cdot$ H. Greisen

Takeda Pharma A/S, Roskilde, Denmark

U. E. Kongsgaard $(\bowtie)$

Department of Anaesthesiology, The Norwegian Radium Hospital, Oslo University Hospital, Montebello 0313, Oslo, Norway

e-mail: ulf.kongsgaard@medisin.uio.no
$<200 \mu \mathrm{g}$ in the majority of patients, requiring only one dose, with no further change in maintenance dose. Pain severity, impact of pain on daily life, and treatment satisfaction significantly improved with Instanyl ${ }^{\mathbb{R}}$ treatment. No unexpected ADRs occurred.

Keywords Cancer · Breakthrough pain · Fentanyl · Instanyl · Intranasal $\cdot$ Observational

\section{Background}

Cancer patients may experience persistent background pain, interspersed with transitory exacerbations of pain that 'breakthrough' the background medication, known as breakthrough pain (BTP). BTP was first defined in 1990 as 'a transitory exacerbation of pain that occurs on a background of otherwise stable pain in a patient receiving chronic opioid therapy' [1]. Many definitions for BTP have since been proposed and are in use but, as yet, no single definition has been widely accepted [2]. The characteristics of individual BTP episodes vary [3-5]; there are reports of median times to reach peak intensity of 3 and $15 \mathrm{~min}$, median durations of 30 and $60 \mathrm{~min}$, and median frequencies of 3 and 6 times per day $[1,6,7]$. BTCP episodes are usually severe $[1,7]$.

BTP may impact on patients' quality of life since it is associated with impairments in daily physical functioning and psychological distress $[6,8]$. BTP places a high economic burden on society and healthcare services $[9,10]$, and may be a significant predictor of increased pain-related costs [11]. Successful management of BTP is an important unmet need in the treatment of cancer patients.

Oral opioids (morphine, oxycodone, and hydromorphine) are commonly used to treat BTP $[12,13]$, but can take $\sim 30$ min to produce an analgesic effect, which then lasts for $\sim 4 \mathrm{~h}$ [12]. While this may be suitable for treating some pain 
conditions, the majority of BTP episodes require a treatment with a fast onset of action and short duration of effect. To improve the treatment of BTP, a range of fast-acting fentanyl formulations have been developed and are approved in this indication. These agents allow rapid absorption and quick onset of effect, with good levels of acceptability to patients [4]. For optimal BTP management, it is recommended that opioids are titrated on an individual patient basis to a successful dose that produces adequate analgesia, with minimal adverse events $[4,14,15]$.

Instanyl ${ }^{\circledR}$ (intranasal fentanyl spray [INFS]) is a novel treatment option approved for the management of BTP in adult cancer patients already receiving maintenance opioid therapy for chronic cancer pain. Clinical studies in cancer patients have shown that INFS provides clinically relevant reductions in pain intensity [16], with superior efficacy and patient preference, compared to oral transmucosal fentanyl citrate (OTFC) [17]. Previous evaluations of Instanyl ${ }^{\circledR}$ were conducted through controlled clinical trials. The aim of the present study is to explore the actual use of Instanyl ${ }^{\circledR}$ and success of titration in real-life settings, and evaluate the impact of Instanyl ${ }^{\circledR}$ on patients' daily life and treatment satisfaction.

\section{Method}

\section{Study design}

A non-interventional, 3-month observational, prospective, cohort study design was employed to follow cancer patients with BTP receiving Instany $1{ }^{\circledR}$ under routine clinical practice. The study was conducted according to the Declaration of Helsinki, Good Pharmacoepidemiological Practices, the Data Protection Directive, and any local requirements. Relevant Independent Ethics Committees granted ethical approval.

Study participation had no impact on the patient (except for the collection of informed consent, requested after the treatment decision); the decision to prescribe Instanyl ${ }^{\circledR}$ was determined according to the summary of product characteristics (SPC) before study entry and as part of current practice, and Instanyl ${ }^{\circledR}$ was not taken until after the baseline visit. Patients were not subjected to additional procedures; participating study physicians were responsible for all decisions regarding patient care.

\section{Patients}

Eligible patients were adult cancer patients (in-/outpatients) suffering from BTP who had not been treated with Instanyl ${ }^{\circledR}$ in the month prior to baseline, and who had been prescribed Instanyl $l^{\circledR}$ in accordance with the SPC (i.e., at marketed doses of 50,100 , or $200 \mu \mathrm{g}$ ) [18]. All patients provided informed consent for the collection of data prior to study inclusion.
Data collection

Patients were recruited from 61 study centres across Denmark, France, Greece, Ireland, Norway, Sweden and the UK. Data were collected at three time points corresponding with patients' usual scheduled visits (not pre-specified by the study protocol) - baseline, Week 4 ( -1 week/ +2 weeks), and Week 13 ( -1 week/ +4 weeks). For patients discontinuing before Week 13, data were collected at the time of discontinuation. Data were mainly obtained from source data, by the investigator, and were collected for Instanyl ${ }^{\circledR}$-treated patients only.

Two patient-rated questionnaires were validated for use in the UK and France - the Brief Pain Inventory-Short Form (BPI-SF) to assess severity of pain and impact of pain on daily life domains, and the Patient Treatment Satisfaction Scale (PTSS) to assess satisfaction with current pain medication. Data from the questionnaires were collected only from those countries in which this is allowed under the concept of a noninterventional study (i.e., UK and France), and from sites where these types of questionnaires are used in standard care. BPI-SF and PTSS scores were assessed at baseline and Week 4 (not at Week 13, as the rapid progression of disease/low completer rate expected in this patient population would likely be a confounding factor by this time point).

\section{Outcome variables}

The primary outcome variables were, success of titration (defined as reaching a maintenance dose) and dose level of Instanyl ${ }^{\circledR}$ after titration (defined as the maintenance dose reached).

Secondary outcome variables included, change in the maintenance dose of Instanyl ${ }^{\circledR}$ and in the level of background pain medication, BPI-SF and PTSS Total and domain scores, adverse drug reactions (ADRs), and reason for, and time to, Instanyl ${ }^{\mathbb{R}}$ termination.

Data analysis

Data from the overall analysis set (all patients enrolled in the study, excluding those with data outside the permitted window for visits) were analysed using epidemiological methods. After the study ended, and before data analyses began, visit windows were widened to include more data (a relatively high proportion of visits occurred outside the originally planned visit windows). The Week 4 visit window increased by 1 day to -1 week/+2 weeks and 1 day; the Week 13 visit window was revised to include any data collected from Day 74 to study end.

Planned recruitment was $300-1,000$ patients, allowing the proportion of successfully titrated patients to be estimated within 3.1-5.7\% with $95 \%$ confidence. An ADR that was expected in one of 100 patients would be observed at least 
Table 1 Patient demographics and baseline characteristics (overall analysis set, $N=309$ )

\begin{tabular}{|c|c|}
\hline Mean age, years (SD) & $60(12.8)$ \\
\hline Male, $n(\%)$ & $173(56)$ \\
\hline Mean BMI, kg/m² (SD) & $23.5(4.3)$ \\
\hline \multicolumn{2}{|l|}{ Site of primary tumour, $n(\%)$} \\
\hline Lung/respiratory system & $78(25.2)$ \\
\hline Colon/rectal system & $41(13.3)$ \\
\hline Breast & $32(10.4)$ \\
\hline Gastro-oesophageal system & $19(6.1)$ \\
\hline Head and neck & $18(5.8)$ \\
\hline Prostate & $18(5.8)$ \\
\hline Pancreas & $17(5.5)$ \\
\hline Female genital & $16(5.2)$ \\
\hline Urological & $16(5.2)$ \\
\hline Unknown primary tumour & $14(4.5)$ \\
\hline Haematological & $12(3.9)$ \\
\hline Musculoskeletal system & $6(1.9)$ \\
\hline Central nervous system & $1(0.3)$ \\
\hline Liver & $0(0.0)$ \\
\hline Other & $21(6.8)$ \\
\hline \multicolumn{2}{|l|}{ Metastases present, $n(\%)$} \\
\hline Overall & $263(85.1)$ \\
\hline In bone & $162(52.4)$ \\
\hline In areas other than bone ${ }^{a}$ & $214^{\mathrm{a}}(69.3)$ \\
\hline \multicolumn{2}{|l|}{ Episodes of BTP per day, $n(\%)$} \\
\hline$<1$ & $22(7.1)$ \\
\hline $1-2$ & $74(23.9)$ \\
\hline $3-4$ & $132(42.7)$ \\
\hline$>4$ & $81(26.2)$ \\
\hline \multicolumn{2}{|l|}{ Usual duration of BTP episodes, $n(\%)$} \\
\hline$<30 \mathrm{~min}$ & $87(28.2)$ \\
\hline $30-60 \mathrm{~min}$ & $154(49.8)$ \\
\hline$>60 \mathrm{~min}$ & $60(19.4)$ \\
\hline$<1 \mathrm{~h}^{\mathrm{b}}$ & $6(1.9)$ \\
\hline Not recorded & $2(0.6)$ \\
\hline \multicolumn{2}{|l|}{ Localisation of BTP, $n(\%)$} \\
\hline At site of primary tumour & $121(39.2)$ \\
\hline At site of metastases & $215(69.6)$ \\
\hline Other & $37(12.0)$ \\
\hline Patients reported to be receiving treatment for BTP, $n(\%)$ & $192(62.1)$ \\
\hline \multicolumn{2}{|l|}{ Current BTP treatment, $n(\%)$} \\
\hline Fentanyl $^{\mathrm{c}}$ & $18(5.8)$ \\
\hline Morphine $^{\mathrm{d}}$ & $75(24.3)$ \\
\hline PCA pump & $1(0.3)$ \\
\hline Oxycodone/oxycodone hydrochloride & $93(30.1)$ \\
\hline Ketobemidone/ketobemidone hydrochloride & $10(3.2)$ \\
\hline Other & $15(4.9)$ \\
\hline None & $117(37.9)$ \\
\hline Patients receiving background medication, $n(\%)$ & $309(100.0)$ \\
\hline
\end{tabular}

Table 1 (continued)

\begin{tabular}{ll}
\hline Strong opioids & \\
Fentanyl & $184(59.5)$ \\
Morphine & $46(14.9)$ \\
Hydromorphone & $2(0.6)$ \\
Oxycodone & $82(26.5)$ \\
Other & $19(6.1)$ \\
Weak opioid & $23(7.4)$ \\
Non-opioid & $12(3.9)$ \\
Adjuvant drug & $11(3.6)$ \\
Other treatment & $1(0.3)$ \\
Mean level of background pain medication (morphine equivalent, \\
mg/day) (SD) \\
Strong opioid \\
Fentanyl \\
Morphine & $156(194.6)$ \\
Hydromorphone & $419(1,597.5)$ \\
Oxycodone & $240(248.9)$ \\
Other & $205(237.6)$ \\
Weak opioid & $253(321.4)$ \\
\end{tabular}

$N$ number of patients, $n$ number of patients with event, $S D$ standard deviation, $B M I$ body mass index, $B T P$ breakthrough pain, $P C A$ patientcontrolled analgesia

${ }^{a}$ Of these, 113 patients also had metastases present in bone

${ }^{\mathrm{b}}$ Category was used prior to a protocol amendment. However, it can be seen that $247(79.9 \%)$ patients were experiencing BTP with a usual duration of $60 \mathrm{~min}$ or less

${ }^{\mathrm{c}}$ Includes oral transmucosal fentanyl citrate, $n=5(1.6 \%)$; fentanyl buccal tablet, $n=3$ (1.0 \%); sublingual fentanyl, $n=10(3.2 \%)$

${ }^{\mathrm{d}}$ Includes oral morphine, $n=53(17.2 \%)$ and other forms of morphine, $n=22(7.1 \%)$

${ }^{\mathrm{e}}$ Some patients were taking $\geq 1$ type of medication for background pain

once in 300 to 1,000 patients dosed with Instanyl ${ }^{\circledR}$, with a probability of $0.95-0.99996$.

Descriptive analyses were provided for all collected data. For the primary outcome variables, the proportion of patients with successful titration was calculated (with $95 \%$ confidence intervals [CIs]) using the number of patients with a baseline visit as the denominator; the proportions of patients titrated to the different dose levels $(50,100$, and $200 \mu \mathrm{g})$ were calculated using the number of patients with successful titration as the denominator. The primary outcome variable was examined across levels of baseline characteristics - age, gender, body mass index (BMI), type of cancer (including presence and site of metastases), BTP episodes by frequency, duration and localisation, current BTP medication, and current background pain medication - multivariate logistic regression adjusted for potential confounders of any associations.

For the secondary efficacy variables, the change in maintenance dose from Week 4 to Week 13 was analysed for patients reaching a maintenance dose. Since a patient's pain could change, the protocol allowed for a dose adjustment (increase or decrease) after the first maintenance dose was achieved at the end of titration. Therefore, the highest prescribed dose level and the usual dose per BTP episode were 
Fig. 1 Success of titration proportion of patients successfully titrated to a maintenance dose of Instanyl ${ }^{\circledR}$ $(50,100$ or $200 \mu \mathrm{g})$ across seven European countries. Doses represent the first maintenance dose level of Instanyl ${ }^{\circledR}$ achieved at the end of titration

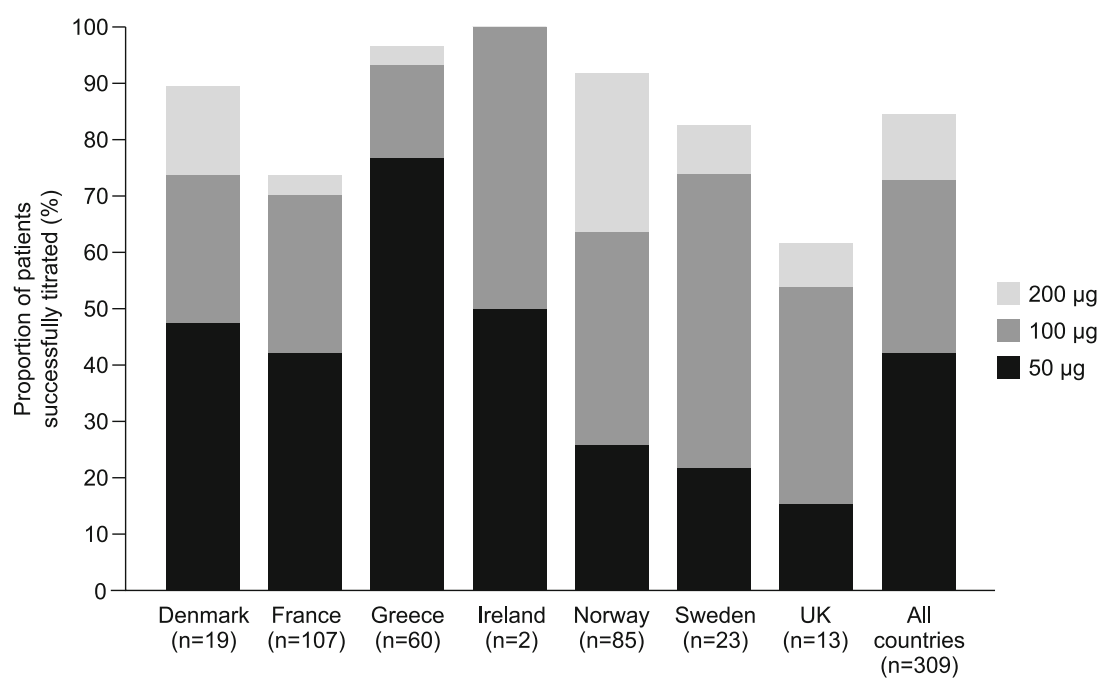

also recorded. The change in the level of background pain medication (expressed as morphine equivalents) was analysed from baseline to Week 4 and to Week 13, and from Week 4 to Week 13. BPI-SF and PTSS Total and domain scores (for UK and France) were summarised at baseline and Week 4; the change from baseline to Week 4 was analysed using a paired $t$ test or a non-parametric Wilcoxon signed-rank test, where appropriate. Incidence rates of ADRs were summarised, as were the primary reasons for terminating Instanyl ${ }^{\circledR}$ treatment. Time to discontinuation of Instanyl ${ }^{\circledR}$ was calculated as the difference in days between baseline and the last known date of Instany $l^{\circledR}$ treatment, plus 1 day, and presented as a KaplanMeier plot (with $95 \% \mathrm{CI}$ ).

For descriptive statistics, no imputation of missing data occurred. Patients lacking data to show whether or not a successful titration had been achieved were considered not to have been successfully titrated. Questionnaire data were treated as missing and excluded from the analyses where patients had not completed total and domain scores for all four pain severity questions and a minimum of four interference questions on the BPI-SF, and if fewer than four of the six questions of the PTSS had been completed.

\section{Results}

The study was initiated on 28 December 2009 and completed on 19 April 2012. In total, 309 patients were enrolled, comprising the overall analysis set; 107 (34.6\%) patients completed the study. Data was collected at baseline for all patients, and within the Week 4 and Week 13 windows for 225 (72.8\%) and 128 (41.1\%) patients, respectively.

Patient demographics and baseline characteristics are summarised in Table 1 . The most common site of primary tumour was the lung/respiratory system and the majority of patients had metastases. Most patients were experiencing 3-4 episodes of BTP per day, with approximately one quarter of patients suffering from $>4$ BTP episodes daily. In the majority of patients, BTP episodes lasted 30-60 min, and originated from the site of metastases. At baseline, 117 (37.9\%) patients were not receiving any BTP treatment. Oral oxycodone or oral morphine was the sole BTP treatment for 80 (26\%) and 46 (15\%) patients, respectively. Fentanyl was most commonly prescribed to treat background pain, followed by oxycodone.

\section{Success of titration}

Excluding Ireland, where only two patients were enrolled, the proportion of patients successfully titrated to a maintenance dose was greatest in Greece and lowest in the UK (Fig. 1). For all countries combined, the majority of patients were successfully titrated to a maintenance dose of $50 \mu \mathrm{g}$, with fewest patients titrated to $200 \mu \mathrm{g}$. A total of $48(15.5 \%)$ patients were not successfully titrated due to patients being withdrawn from ( $n=46$ ), or not completing $(n=2)$, the study.

Greater success of titration was associated with being younger, female, having a higher BMI, and having metastases (Table 2). The logistic regression showed that, after accounting for country differences, a higher success rate was associated with being female (odds ratio $[\mathrm{OR}]=3.6 ; 95 \% \mathrm{CI}: 1.6$, 7.8), having BTP originating from the site of the primary tumour $(\mathrm{OR}=3.1 ; 95 \% \mathrm{CI}: 1.3,7.4)$, and the presence of bone metastases $(\mathrm{OR}=2.2 ; 95 \% \mathrm{CI}: 1.0-4.9)$. However, the overall success rate was not affected when adjusting for these variables.

Maintenance treatment

The median time from baseline to maintenance treatment was 29 days (95 \% CI: 29-31), possibly a reflection of the study 
Table 2 Success of titration by patient demographics and baseline characteristics (overall analysis set, $N=309$ )

\begin{tabular}{|c|c|}
\hline $\begin{array}{l}\text { Patient demographic/baseline } \\
\text { characteristic }\end{array}$ & $\begin{array}{l}\text { Patients successfully titrated, } \\
\mathrm{n}(\%)\end{array}$ \\
\hline Total & $261 / 309(84.5)$ \\
\hline \multicolumn{2}{|l|}{ Age (years) } \\
\hline$<60$ & $135 / 152(88.8)$ \\
\hline $60-75$ & $104 / 127(81.9)$ \\
\hline$>75$ & $22 / 30(73.3)$ \\
\hline \multicolumn{2}{|l|}{ Gender } \\
\hline Male & $138 / 173(79.8)$ \\
\hline Female & $123 / 136(90.4)$ \\
\hline \multicolumn{2}{|l|}{ BMI $\left(\mathrm{kg} / \mathrm{m}^{2}\right)$} \\
\hline$<18.5$ (underweight) & $21 / 27(77.8)$ \\
\hline 18.5 to $<25$ (normal weight) & $147 / 174(84.5)$ \\
\hline$\geq 25$ (overweight/obese) & $79 / 90(87.8)$ \\
\hline Missing & $14 / 18(77.8)$ \\
\hline \multicolumn{2}{|l|}{ Presence of metastases } \\
\hline \multicolumn{2}{|l|}{ Overall } \\
\hline Yes & $224 / 263(85.2)$ \\
\hline No & $37 / 46(80.4)$ \\
\hline \multicolumn{2}{|l|}{ In bone } \\
\hline Yes & $140 / 162(86.4)$ \\
\hline No & $121 / 147(82.3)$ \\
\hline \multicolumn{2}{|l|}{ In areas other than bone } \\
\hline Yes & $182^{\mathrm{a}} / 214(85.0)$ \\
\hline No & $79 / 95(83.2)$ \\
\hline \multicolumn{2}{|l|}{ Episodes of BTP per day } \\
\hline$<1$ & $17 / 22(77.3)$ \\
\hline $1-2$ & $61 / 74(82.4)$ \\
\hline $3-4$ & $116 / 132(87.9)$ \\
\hline$>4$ & $67 / 81(82.7)$ \\
\hline \multicolumn{2}{|l|}{ Usual duration of BTP episodes } \\
\hline$<30 \mathrm{~min}$ & $76 / 87(87.4)$ \\
\hline $30-60 \mathrm{~min}$ & $133 / 154(86.4)$ \\
\hline$>60 \mathrm{~min}$ & $48 / 60(80.0)$ \\
\hline$<1 \mathrm{~h}^{\mathrm{b}}$ & $4 / 6(66.7)$ \\
\hline vNot recorded & $0 / 2(0.0)$ \\
\hline \multicolumn{2}{|l|}{ Localisation of BTP } \\
\hline From site of primary tumour & $107 / 121(88.4)$ \\
\hline From site of metastases & $183 / 215(85.1)$ \\
\hline Other & $30 / 37(81.1)$ \\
\hline \multicolumn{2}{|l|}{ Currently treated for BTP } \\
\hline Yes & $158 / 192(82.3)$ \\
\hline No & $103 / 117(88.0)$ \\
\hline
\end{tabular}

Data are presented as $n(\%)$

$N$ number of patients, $n$ number of patients with event, $B M I$ body mass index, $B T P$ breakthrough pain.

${ }^{a}$ Of these, 113 patients also had metastases present in bone

${ }^{\mathrm{b}}$ Category was used prior to a protocol amendment. However, it can be seen that $213(68.9 \%)$ patients were experiencing BTP with a usual duration of $60 \mathrm{~min}$ or less design (i.e., investigators first determined the maintenance dose at Week 4). The most common first maintenance dose achieved was $50 \mu \mathrm{g}$, followed by $100 \mu \mathrm{g}$, then $200 \mu \mathrm{g}$; a similar pattern was observed for the highest prescribed dose level achieved (Table 3). The usual dose per BTP episode was 50 or $100 \mu \mathrm{g}$ in the majority of patients (Table 3$)$. Most patients $(70 \%)$ received one dose per BTP episode (50 $\mu \mathrm{g}$ : $70 \%$; $100 \mu \mathrm{g}$ : $73 \%$; $200 \mu \mathrm{g}$ : $64 \%)$. The majority showed no change in maintenance dose from Week 4 to Week 13 (Table 4).

Background medication

There was little evidence of a change in background pain medication (as morphine equivalent) from baseline to Week 4 (median change $=0 \mathrm{mg} /$ day; inter-quartile range, Q1-Q3: 060 ) or from Week 4 to Week 13 (median change $=0 \mathrm{mg} /$ day; Q1-Q3: 0-77), but a slight increase from baseline to Week 13 (median change $=40 \mathrm{mg} /$ day; Q1-Q3: 0-120). No relationship between the successfully titrated dose of Instanyl ${ }^{\circledR}$ and level of background medication was found.

Duration of Instanyl $^{\circledR}$ treatment

At Week 4, $172(55.7 \%)$ patients were treated with Instanyl ${ }^{\circledR}$, falling to $103(33.3 \%)$ patients at Week 13. The median duration of Instanyl ${ }^{\circledR}$ treatment (baseline to end of treatment) was 50 days. The majority of treatment terminations were due to the patients having died $(n=72 ; 23.3 \%)$, which was expected given the underlying disease (no deaths were reported as fatal ADRs). For patients who had not died, the main reasons for termination were lack of efficacy $(n=47$; $15.2 \%)$, and 'other' reasons $(n=46 ; 14.9 \%$; including six $(1.9 \%)$ patients who reported having no more BTP). Of those patients terminating due to lack of efficacy, 14 (4.5\%) were titrated to $50 \mu \mathrm{g}$, seven $(2.3 \%)$ were titrated to $100 \mu \mathrm{g}$, and four $(1.3 \%)$ were titrated to $200 \mu \mathrm{g}$; the remaining $22(7.1 \%)$ patients terminated before a successful titration. An inability to administer drug accounted for Instanyl ${ }^{\circledR}$ termination in 20 $(6.5 \%)$ patients, with the fewest terminations reported in patients with an $\operatorname{ADR}(n=7 ; 2.3 \%)$.

Pain and treatment satisfaction

There was a significant reduction in BPI-SF Total score, and in the domain scores for severity of pain and interference with daily activities, from baseline to Week 4; worst pain scores also significantly improved (Table 5). There was a significant increase in PTSS total score from baseline to Week 4 (Table 5).

Adverse drug reactions

ADRs were extracted from the international drug safety database using the cut-off date of 26 April 2012. By this time, a 
Table 3 Dose level of Instanyl ${ }^{\circledR}$ after successful titration

\begin{tabular}{|c|c|c|c|c|c|}
\hline & \multicolumn{5}{|c|}{ Number of patients successfully titrated (\%) } \\
\hline & $50 \mu \mathrm{g}$ & $100 \mu \mathrm{g}$ & $200 \mu \mathrm{g}$ & $150-200 \mu \mathrm{g}$ & $>200 \mu \mathrm{g}$ \\
\hline First maintenance dose level achieved & $130(49.8)$ & $95(36.4)$ & $36(13.8)$ & - & - \\
\hline Highest prescribed dose level achieved & 119 (45.6) & $97(37.2)$ & $44(16.9)$ & - & - \\
\hline Usual dose per BTP episode $^{a}$ & $82(31.4)$ & $103(39.5)$ & - & $55(21.1)$ & $19(7.3)$ \\
\hline
\end{tabular}

Data based on the total number of patients who were successfully titrated $(N=261)$

$N$ number of patients, $B T P$ breakthrough pain

${ }^{a}$ Reflects the total dose taken per episode (i.e., the prescribed dose $\mathrm{x}$ the number of times the dose was taken, e.g., $50 \mu \mathrm{g}$ taken three times gives a usual dose of $150 \mu \mathrm{g}$ )

total of 18 ADRs had been reported for nine $(2.9 \%)$ patients. Of these, three $(1.0 \%)$ patients reported serious ADRs. ADRs were considered to be related to Instanyl ${ }^{\circledR}$ for seven $(2.3 \%)$ patients (six [1.9\%] patients with non-serious ADRs; one $[0.3 \%]$ patient with serious ADRs). No fatal ADRs were reported. All patients recovered from their ADRs; all, but one, patient discontinued the study early.

\section{Discussion}

Despite various fast-acting fentanyl formulations being available for the treatment of BTP, evidence suggests that their use is not widely recognised $[7,19]$. For example, a recent European survey reports that $38.4 \%$ of oncology nurses are unaware that medications specific for BTP exist; $57 \%$ stated that oral morphine was normally prescribed for BTP [13].

The present study aimed to evaluate the actual use of Instanyl ${ }^{\circledR}$ and success of titration in real-life settings during the early post-launch phase in participating countries, outside the restrictions of a clinical trial. The low number of patients recruited in the study may partly be due to Instanyl ${ }^{\circledR}$ being used in a more carefully selected patient population during this period (i.e., patients with problematic BTP that could not be sufficiently addressed by previously available treatment options, and/or those more positive to new treatments).

Table 4 Change in Instanyl ${ }^{\circledR}$ maintenance dose strength

\begin{tabular}{llllll}
\hline & & \multicolumn{2}{l}{ Week 13 } & \multirow{2}{*}{ Total } \\
\cline { 3 - 4 } & & $50 \mu \mathrm{g}$ & $100 \mu \mathrm{g}$ & $200 \mu \mathrm{g}$ & \\
\hline Week 4 & $50 \mu \mathrm{g}$ & $32(30.5)$ & $15(14.3)$ & $6(5.7)$ & $53(50.5)$ \\
& $100 \mu \mathrm{g}$ & $4(3.8)$ & $23(21.9)$ & $11(10.5)$ & $38(36.2)$ \\
& $200 \mu \mathrm{g}$ & $2(1.9)$ & $0(0.0)$ & $12(11.4)$ & $14(13.3)$ \\
& Total & $38(36.2)$ & $38(36.2)$ & $29(27.6)$ & $105(100.0)$ \\
\hline
\end{tabular}

Data presented as $n(\%)$, based on the total number of patients with data at Week 4 and Week $13(N=105)$

$N$ number of patients, $n$ number of patients with event
The study recruited a patient population with a high presence of metastases all receiving medication for background pain. Most patients were experiencing multiple daily episodes of BTP and were receiving treatment for BTP at baseline. The observation that some patients were experiencing fewer than one, or more than four, BTP episodes daily highlights the need for a treatment strategy tailored to the individual patient. Data collected also indicated that some patients were receiving conventional oral opioids (morphine or oxycodone) alone for BTP, suggesting less than optimal pain management. The high number of patients who died during the study reflected the disease severity; indeed, this was the main reason reported for Instanyl ${ }^{\circledR}$ treatment termination. Lack of efficacy was cited as the main reason for terminating Instanyl ${ }^{\circledR}$ among patients who had not died, possibly because these patients were not titrated to the highest dose. However, this is merely speculation, since the exact reasons cannot be determined.

Overall, successful titration was high in this study $(84.5 \%)$. Although a direct comparison to existing data from clinical trials cannot be made, success rates of approximately $70 \%$ have been reported for fast-acting fentanyl formulations [20-22]. The differences in success of titration based on

Table 5 Change in BPI-SF and PTSS scores

\begin{tabular}{lllll}
\hline & $\begin{array}{l}\text { Absolute } \\
\text { change from } \\
\text { baseline to } \\
\text { Week 4 }\end{array}$ & $p$ value & $\begin{array}{l}\text { Percentage } \\
\text { change from } \\
\text { baseline to } \\
\text { Week 4 }\end{array}$ & $p$ value \\
\hline BPI-SF total score & $-1.0(2.1)$ & $<0.001$ & $-10.2(57.9)$ & $<0.001$ \\
$\begin{array}{l}\text { Severity of pain } \\
\text { Interference with }\end{array}$ & $-0.8(2.0)$ & $<0.001$ & $-10.6(45.6)$ & 0.009 \\
$\begin{array}{l}\text { daily activities } \\
\text { Worst pain score }\end{array}$ & $-1.2(2.5)$ & $<0.001$ & $-3.8(103.6)$ & $<0.001$ \\
PTSS Total score & $14.4(20.9)$ & $<0.001$ & $-12.8(42.7)$ & $<0.001$ \\
\hline
\end{tabular}

Data presented are mean (SD), based on questionnaires administered to patients in the UK and France: BPI-SF, $N=126$; PTSS, $N=37$

BPI-SF Brief Pain Inventory - Short Form, PTSS Patient Treatment Satisfaction Scale, $S D$ standard deviation, $U K$ United Kingdom, $N$ number of patients 
patient characteristics were minor and considered of less importance for clinical practice. Successful titration was highest in Greece and Norway ( $>90 \%$ ), and lowest in the UK and France $(<75)$. It is difficult to explain the observed differences between countries, and the reasons provided here are merely speculative. For example, the high success rate seen in Norway may be due to the high proportion of patients titrated to $200 \mu \mathrm{g}$. However, this theory does not support the high success rate observed in Greece, where the majority of patients were successfully titrated to $50 \mu \mathrm{g}$. Evaluation of patient characteristics did not offer any further potential explanations for the inter-country differences observed. Indeed, success rates may be influenced by more factors than were measured in this study.

Of the patients who were successfully titrated, the majority achieved this with the lowest dose of Instanyl ${ }^{\circledR}(50 \mu \mathrm{g})$, possibly reflecting the high bioavailability observed for intranasal fentanyl [23], and supporting titration from the lowest dose. The observation that most patients achieved successful titration with only one dose of Instanyl ${ }^{\mathbb{R}}$ has positive implications for BTP management.

Given the disease characteristics of the patients recruited, disease progression may have been expected, which would have required an increase in the Instanyl ${ }^{\circledR}$ dose. In fact, the majority of patients showed no change in the maintenance strength dose throughout the study, indicating the effectiveness of Instanyl ${ }^{\circledR}$ in treating BTP. A lack of relationship between the maintenance dose achieved and the level of background pain medication is consistent with other trials involving Instanyl ${ }^{\circledR}$ (unpublished data) and OTFC [24].

Assessments of pain and its impact on daily life showed reductions in pain severity $(11 \%)$, interference with daily activities $(4 \%)$, and worst pain score $(13 \%)$ with Instanyl ${ }^{\circledR}$ that are considered to be highly clinically relevant in a population of patients for whom pain intensity would be expected to increase. Patients' satisfaction with treatment increased during the treatment period. These results indicate the effectiveness of Instanyl ${ }^{\circledR}$ in a patient population with a high probability of disease progression.

As with any study, there are limitations to consider. Firstly, in some countries, patient numbers were small. Secondly, the low number of ADRs reported suggests that patients in such a population (i.e., terminally ill cancer patients receiving treatment for their pain) do not report adverse events due to the disease severity. Indeed, the common side effects of fentanyl can be confused with the side effects arising from a patient's background opioid medication. Thirdly, the lack of a comparator arm may be seen as a further limitation. However, including a comparator arm (placebo or other reference drug) would not have contributed to the main objective of describing the real-life use of Instanyl ${ }^{\circledR}$ for treating BTP in cancer patients. Another limiting factor is that although the overall amount of pain medication was determined before and after initiation of
Instany $l^{\circledR}$ treatment, as there was no placebo control the effect of Instanyl ${ }^{\circledR}$ could not be differentiated from that of existing treatment. Oral morphine and other pain medications are used to treat both background pain and BTP, and these effects cannot be separated. Furthermore, the need for pain treatment in the individual patient may change during the study. Finally, although not a limitation per se, it is a common observation of many studies that simply being enrolled into a trial can influence a patient's perception of treatment satisfaction [25].

In conclusion, the study has shown that in a real-life group of patients with disease progression, the majority achieved a successful titration with Instanyl ${ }^{\circledR}$ at doses below $200 \mu \mathrm{g}$; over one-third of patients were successfully treated with the lowest dose of $50 \mu \mathrm{g}$. The majority of patients were successfully titrated with one dose of Instanyl ${ }^{\circledR}$ and required no further change to their maintenance dose. The severity of pain and associated impact on daily life significantly improved with Instanyl ${ }^{\circledR}$ treatment, as did the level of patient satisfaction with treatment. No unexpected adverse reactions occurred with Instanyl ${ }^{\circledR}$ treatment.

Acknowledgements The authors thank the investigators involved in the study. Assistance with preparing the manuscript was provided by Cambridge Medical Communication Ltd., funded by the study Sponsor, Takeda.

Conflict of interest U. Kongsgaard has received honoraria from Takeda. M. Eeg and H. Greisen are employees of Takeda. The authors have full control of all primary data, and have agreed to allow the journal to review these data if requested.

Open Access This article is distributed under the terms of the Creative Commons Attribution Noncommercial License which permits any noncommercial use, distribution, and reproduction in any medium, provided the original author(s) and the source are credited.

\section{References}

1. Portenoy RK, Hagen NA (1990) Breakthrough pain: definition, prevalence and characteristics. Pain 41:273-281

2. Haugen DF, Hjermstad MJ, Hagen N, Caraceni A, Kaasa S, European Palliative Care Research Collaborative (EPCRC) (2010) Assessment and classification of cancer breakthrough pain: a systematic literature review. Pain 149:476-482

3. Portenoy RK (1997) Treatment of temporal variations in chronic cancer pain. Semin Oncol 24(5 Suppl 16):S16-7-12

4. Zeppetella G (2011) Breakthrough pain in cancer patients. Clin Oncol (R Coll Radiol) 23:393-398

5. Caraceni A, Bertetto O, Labianca R, for the Breakthrough/Episodic Pain Italian Study Group et al (2012) Episodic (breakthrough) pain prevalence in a population of cancer pain patients. Comparison of clinical diagnoses with the QUDEI-Italian questionnaire for intense episodic pain. J Pain Symptom Manage 43:833-841

6. Portenoy RK, Payne D, Jacobsen P (1999) Breakthrough pain: characteristics and impact in patients with cancer pain. Pain 81:129-134

7. Davies A, Zeppetella G, Andersen S et al (2011) Multi-centre European study of breakthrough cancer pain: pain characteristics 
and patient perceptions of current and potential management strategies. Eur J Pain 15:756-763

8. Caraceni A, Martini C, Zecca E, Portenoy RK, for the Working Group of the IASP Task Force on Cancer Pain (2004) Breakthrough pain characteristics and syndromes in patients with cancer pain. An international survey. Palliat Med 18:177-183

9. Fortner BV, Okon TA, Portenoy RK (2002) A survey of pain-related hospitalizations, emergency department visits, and physician office visits reported by cancer patients with and without history of breakthrough pain. J Pain 3:38-44

10. Abernethy AP, Wheeler JL, Fortner BV (2008) A health economic model of breakthrough pain. Am J Manag Care 14:S129-S140

11. Fortner BV, Demarco G, Irving G, Ashley J, Keppler G, Chavez J, Munk J (2003) Description and predictors of direct and indirect costs of pain reported by cancer patients. J Pain Symptom Manage 25:9-18

12. Bennett D, Burton AW, Fishman S et al (2005) Consensus panel recommendations for the assessment and management of breakthrough pain: Part 2. Management. Pharm Ther 30:354-361

13. Rustøen T, Geerling JI, Pappa T et al (2013) A European survey of oncology nurse breakthrough cancer pain practices. Eur J Oncol Nurs 17:95-100

14. Hagen NA, Fisher K, Victorino C, Farrar JT (2007) A titration strategy is needed to manage breakthrough cancer pain effectively: observations from data pooled from three clinical trials. J Palliat Med 10:47-55

15. Kleeberg UR, Filbet M, Zeppetella G (2010) Fentanyl buccal tablet for breakthrough cancer pain: why titrate? Pain Pract 11:185-190

16. Kress HG, Orońska A, Kaczmarek Z, Kaasa S, Colberg T, Nolte T (2009) Efficacy and tolerability of intranasal fentanyl spray 50 to $200 \mu \mathrm{g}$ for breakthrough pain in patients with cancer: a Phase III, multinational, randomized, double-blind, placebo-controlled, crossover trial with a 10month, open-label extension treatment period. Clin Ther 31:1177-1191
17. Mercadante S, Radbruch L, Davies A et al (2009) A comparison of intranasal fentanyl spray with oral transmucosal fentanyl citrate for the treatment of breakthrough cancer pain: an open-label, randomised, crossover trial. Curr Med Res Opin 25:2805-2815

18. Takeda UK Ltd (2013) Instanyl ${ }^{\circledR} 50,100$ and $200 \mathrm{mcg}$ nasal spray, solution in single-dose container. (Summary of product characteristics). Takeda UK Ltd, Buckinghamshire

19. Mercadante S, Villari P, Casuccio A (2011) An Italian survey on the attitudes in treating breakthrough cancer pain in hospice. Support Care Cancer 19:979-983

20. Coluzzi PH, Schwartzberg L, Conroy JD et al (2001) Breakthrough cancer pain: a randomized trial comparing oral transmucosal fentanyl citrate $\left(\mathrm{OTFC}^{\circledR}\right)$ and morphine sulfate immediate release $\left(\mathrm{MSIR}^{\circledR}\right)$. Pain 91:123-130

21. Hanks GW, Nugent M, Higgs CM, Busch MA, OTFC Multicentre Study Group (2004) Oral transmucosal fentanyl citrate in the management of breakthrough pain in cancer: an open, multicentre, dosetitration and long-term use study. Palliat Med 18:698-704

22. Weinstein SM, Messina J, Xie F (2009) Fentanyl buccal tablet for the treatment of breakthrough pain in opioid-tolerant patients with chronic cancer pain: a long-term, open-label safety study. Cancer 115: 2571-2579

23. Foster D, Upton R, Christrup L, Popper L (2008) Pharmacokinetics and pharmacodynamics of intranasal versus intravenous fentanyl in patients with pain after oral surgery. Ann Pharmacother 42:1380 1387

24. Portenoy RK, Payne R, Coluzzi P et al (1999) Oral transmucosal fentanyl citrate (OTFC) for the treatment of breakthrough pain in cancer patients: a controlled dose titration study. Pain 79:303-312

25. Verheggen FW, Nieman FH, Reerink E, Kok GJ (1998) Patient satisfaction with clinical trial participation. Int J Qual Health Care 10:319-330 